\title{
Two-Dimensional Topology Optimization of a Horn Antenna
}

\author{
Huaxu Dan \\ Kingswood Rd, West Hartford, CT, USA \\ Email: danhuaxutommy@gmail.com
}

How to cite this paper: Dan, H.X. (2020) Two-Dimensional Topology Optimization of a Horn Antenna. Open Journal of Optimization, 9, 39-46.

https://doi.org/10.4236/ojop.2020.93004

Received: April 8, 2020

Accepted: July 10, 2020

Published: July 13, 2020

Copyright (C 2020 by author(s) and Scientific Research Publishing Inc. This work is licensed under the Creative Commons Attribution International License (CC BY 4.0).

http://creativecommons.org/licenses/by/4.0/

\begin{abstract}
A two-dimensional horn antenna is used as a model for topology optimization. In order to employ the topology optimization, each point in the domain is controlled by a function which is allowed to take values between 0 and 1 . Each point's distinct value then gives it an effective permittivity, either close to that of polyimide or that of air, two materials considered in this study. With these settings, the optimization problem becomes finding the optimal distribution of materials in a given domain, and is solved under constraints of reflection and material usage by the Method of Moving Asymptotes. The final configuration consists of two concentric arcs of air while polyimide takes up the rest of the domain, a result relatively unsensitive to the choice of constraints and initial values. Compared to the unoptimized antenna, a slimmer main lobe is observed and the gain boosts.
\end{abstract}

\section{Keywords}

Topology Optimization, Horn Antenna, Material

\section{Introduction}

Antennas are devices acting as a transition between the free space and the power source [1] [2]. They play an important role in wireless communication, where these devices transmit and receive signals from afar. In their long history, many types of antennas have been developed and constructed over nearly a century [3]-[8]. Among them, horn antennas are built to achieve high gain in frequencies above very high frequency.

Topology optimization is a method that optimizes material layout within a given design space, under a set of constraints. In order to generate the optimal topologies, microstructures, composites of material and void, are employed to form the domain. Therefore, the topology optimization problem in fact turns 
into a material distribution problem, whose calculation requires considerably less computing costs. Typical algorithm employed is either gradient-based methods such as the optimality criteria algorithm and the method of moving asymptotes (MMA) or non-gradient-based algorithms such as particle swarm or genetic algorithms. Topology optimization has a wide range of applications in aerospace, mechanical, bio-chemical and civil engineering [9] [10].

In this work, we adopt topology optimization method for designing a two-dimensional horn antenna.

Optimization results show that a highly directive antenna with non-intuitive materials distribution can be obtained with this powerful method, opening new ways for future antenna design.

\section{Background and Model Definition}

In electromagnetics, Maxwell's equations govern. Together with proper boundary conditions, the radiation pattern of antennas can be solved.

The simulation implemented in this paper seeks to find an optimal material distribution for a two-dimensional horn antenna in order to reach a high gain. Leaving the geometry unchanged, the material configuration can be another angle for antenna design and whose optimization might improve the antenna's performances, particularly its gain as being discussed in this paper.

In this model, every point in the domain of the horn is assigned a $\eta$ variable, whose value is to be changed by the optimization. $\eta$ determines the permittivity, or equivalently what material is used, at one point according to the $\varepsilon_{\text {ramp }}$ function. $\eta$ ranges from 0 to 1 ; when $\eta$ decreases from 0.5 , the value of $\varepsilon_{\text {ramp }}$ reduces rapidly to 1 , suggesting a usage of air; when $\eta$ increases from 0.5 , the value of $\varepsilon_{\text {ramp }}$ rises sharply to 3.5 , implying a usage of polyimide (PI). $\varepsilon_{\text {ramp }}$ as a function of $\eta$ is shown in Equation (1). When the $\eta$ value at one point equals to 0.5 , it is saying that a composite of $50 \%$ air and $50 \%$ polyimide is used. However, in practice this sort of composite may be difficult to comprehend or implement. A definite usage of material is preferred. To ensure that most points are assigned a $\eta$ value away from ambiguity, a lower bound of the average of all points' Weight function's value is applied to the domain in the optimization. Weight function yields a large value when $\eta$ at that specific point is very different from 0.5 , whereas when $\eta$ is close to 0.5 , it contributes negligibly to the congregation. The graphs of the $\varepsilon_{\text {ramp }}$ and Weight functions are shown in Figure 1 and Figure 2, respectively. The reason why only permittivity changed is that both permeability and conductivity of air and polyimide are nearly equal. All parameters used in this work are listed in Table 1.

$$
\begin{gathered}
\varepsilon_{\text {ramp }}=\varepsilon_{\text {air }}+\frac{\varepsilon_{\text {polymide }}-\varepsilon_{\text {air }}}{1+\left[1+e^{-3 p 1(\eta-0.5)}\right]} \\
\text { Weight }=1+\frac{1}{1+e^{-7 p(\eta-0.68)}}-\frac{1}{1+e^{-7 p(\eta-0.32)}}
\end{gathered}
$$




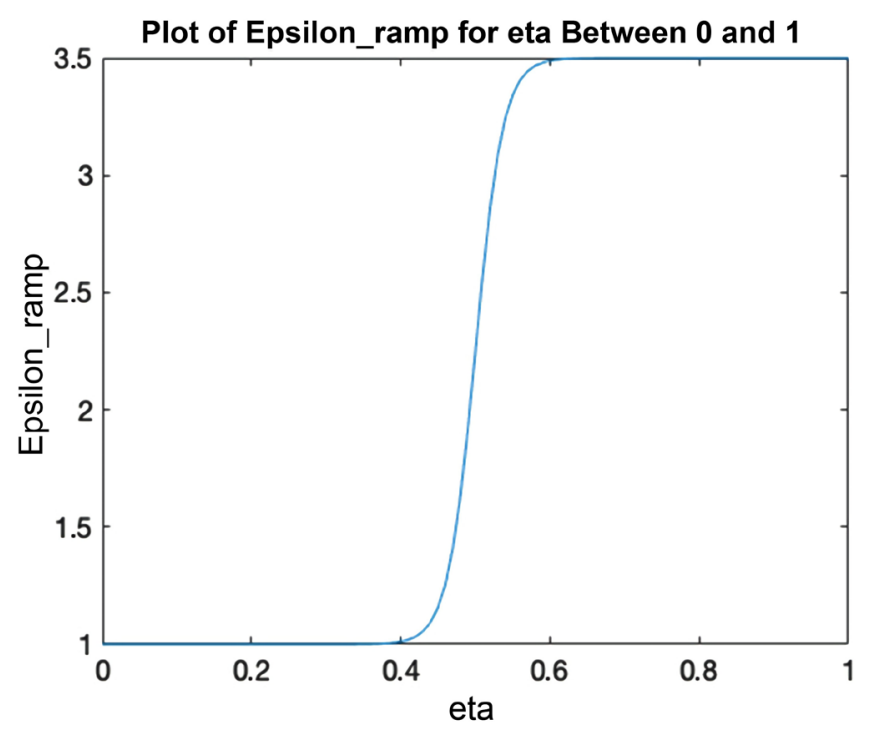

Figure 1. The graph of function $\varepsilon_{\text {ramp }}$.

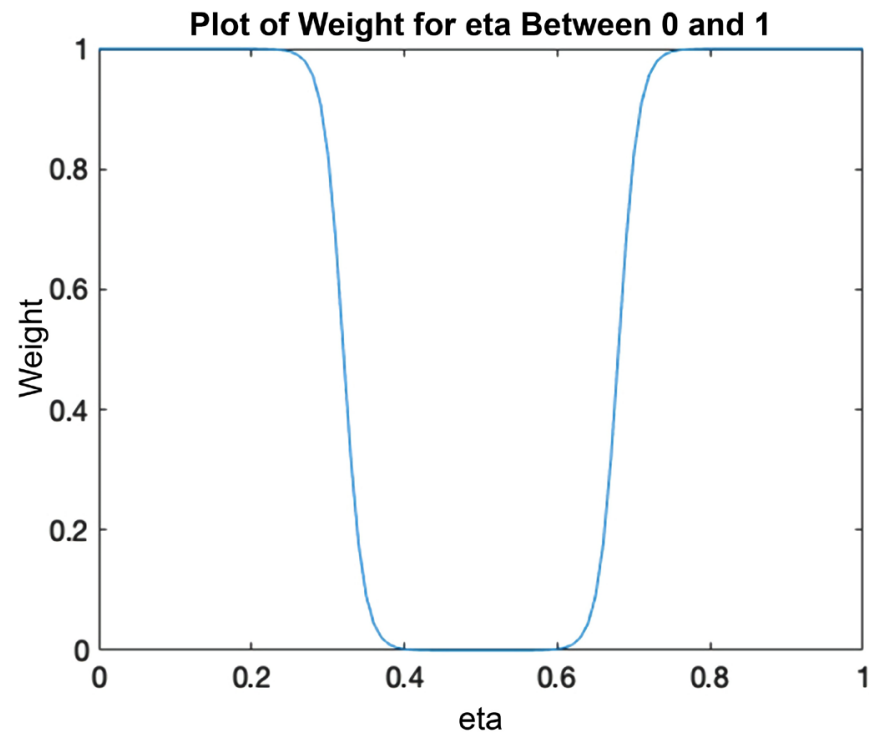

Figure 2. The graph of function weight.

Table 1. Parameters used in this study.

\begin{tabular}{ccc}
\hline$\eta_{\text {initial }}$ & 0.4 & initial value of $\eta$ \\
\hline$f_{0}$ & $5[\mathrm{GHz}]$ & operating frequency \\
wavelength & $c_{0} / f_{0}$ & wavelength under the specified frequency \\
$p_{1}$ & 18 & parameter in $\varepsilon_{\text {ramp }}$ \\
$p$ & 11 & parameter in Weight \\
$\varepsilon_{\mathrm{PI}}$ & 3.5 & relative permittivity of material polyimide \\
$\varepsilon_{\text {air }}$ & 1 & relative permittivity of material air \\
$L_{\text {antenna }}$ & $c_{0} / f_{0}$ & length of the antenna \\
$r_{\text {air }}$ & $1.5 L_{\text {antenna }}$ & radius of the calculated far field filled with air \\
\hline
\end{tabular}




\section{Continued}

\begin{tabular}{|c|c|c|}
\hline$r_{\text {integration }}$ & $0.9 r_{\text {air }}$ & radius of the integral arc \\
\hline$\alpha$ & $30^{\circ}$ & sector angle of the integral line \\
\hline$L_{\text {integration }}$ & $\alpha / 180 \pi r_{\text {integration }}$ & length of the line integration \\
\hline$W_{\text {port }}$ & $\mathrm{L}_{\text {antenna }} / 20$ & width of the port \\
\hline$L_{\text {port }}$ & $5 W_{\text {port }}$ & length of the transmission part \\
\hline$Z_{\text {char }}$ & $50[\mathrm{ohm}]$ & characteristic impedance of the port \\
\hline$D_{\mathrm{PML}}$ & $c_{0} / 2 f_{0}$ & thickness of the perfectly matched layer \\
\hline Area & $\sqrt{3}\left(L_{\text {integration }}\right)^{2} / 4$ & area of the "horn" \\
\hline
\end{tabular}

$$
\begin{aligned}
\text { Objective } & =\frac{2 \pi r_{\text {integration }} \int_{A}^{B} E_{\text {normalized }}(\theta) \mathrm{d} \theta}{L_{\text {integration }} \oint E_{\text {normalized }}(\theta) \mathrm{d} \theta} \\
\text { Reflection } & =(S 11)^{2}
\end{aligned}
$$

The layout of the antenna and its corresponding simulation region is shown in Figure 3. A perfectly matched layer with circular shape surrounds the entire simulation region to eliminate any reflection that could occur at the outer boundary.

The optimization objective expressed in Equation (3) is defined as the ratio of the radiation intensity in the far field at one particular direction to the average radiation intensity of an isotropic antenna: the linear integration of the normalized far field strength over the arc $\mathrm{AB}$ over the linear integration of the normalized far field strength over the entire circle, on which the arc $\mathrm{AB}$ lies, namely the total power. In the optimization, the Reflection is given an upper bound of 0.45 , because excessive reflection from the hardware not only influences the power output, but can also damage the antenna and the transmitting line in practice. A uniform lumped port is used on arc $\mathrm{CD}$ to feed the antenna. Perfect electric conductor is applied to CE, DF, EG and FH, as shown in Figure 3. Finally, outside the antenna is defined as the far field, with the outermost layer being the perfectly matched layer to absorb radiation. Figure 4 depicts the mesh of the model. The Optimization module is set to find the maximum of the variable Objective, using the Method of Moving Asymptotes.

\section{Results and Discussions}

The final electric field norm and $\eta$ distribution given by the topology optimization are shown in Figure 5. Particularly, on the right side, dark color denotes a low $\eta$ value, indicating at that point, the permittivity is the permittivity of air. On the contrary, light color suggests a high $\eta$ value, giving the point the permittivity of PI. In the horn, bands of air and PI appear alternately. Notably, dark points scatter in bands of air. The optimized results are quite non-intuitive, indicating the power of topology optimization.

Figures 6 to 8 show three variables as a function of iteration number during 


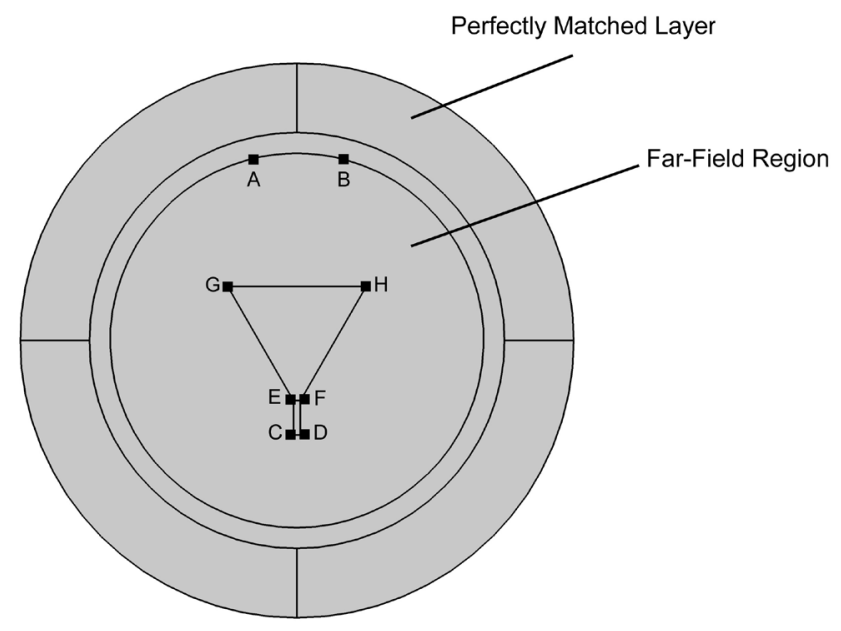

Figure 3. Schematic of the antenna and outer simulation area under study.

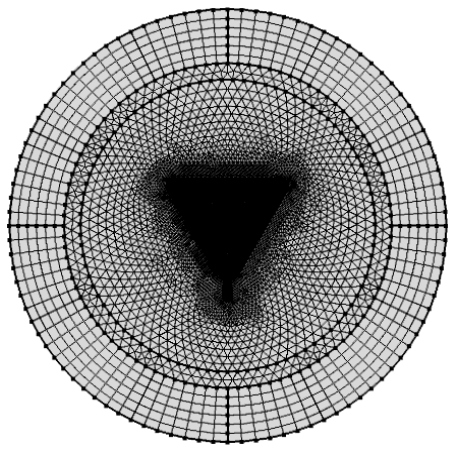

Figure 4. Meshing for finite element method simulation. The antenna region is intentionally fine meshed to give better resolution.

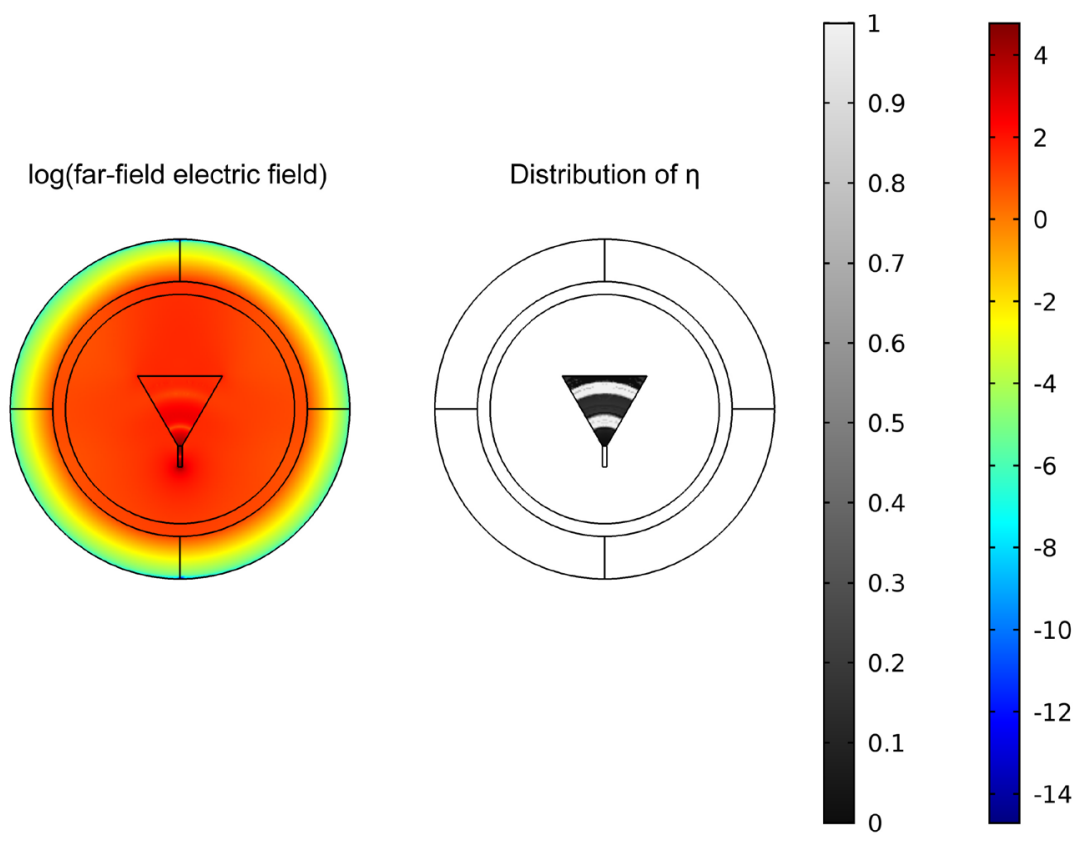

Figure 5. Electromagnetic field distribution (left) and $\eta$ value (right) after topology optimization. 


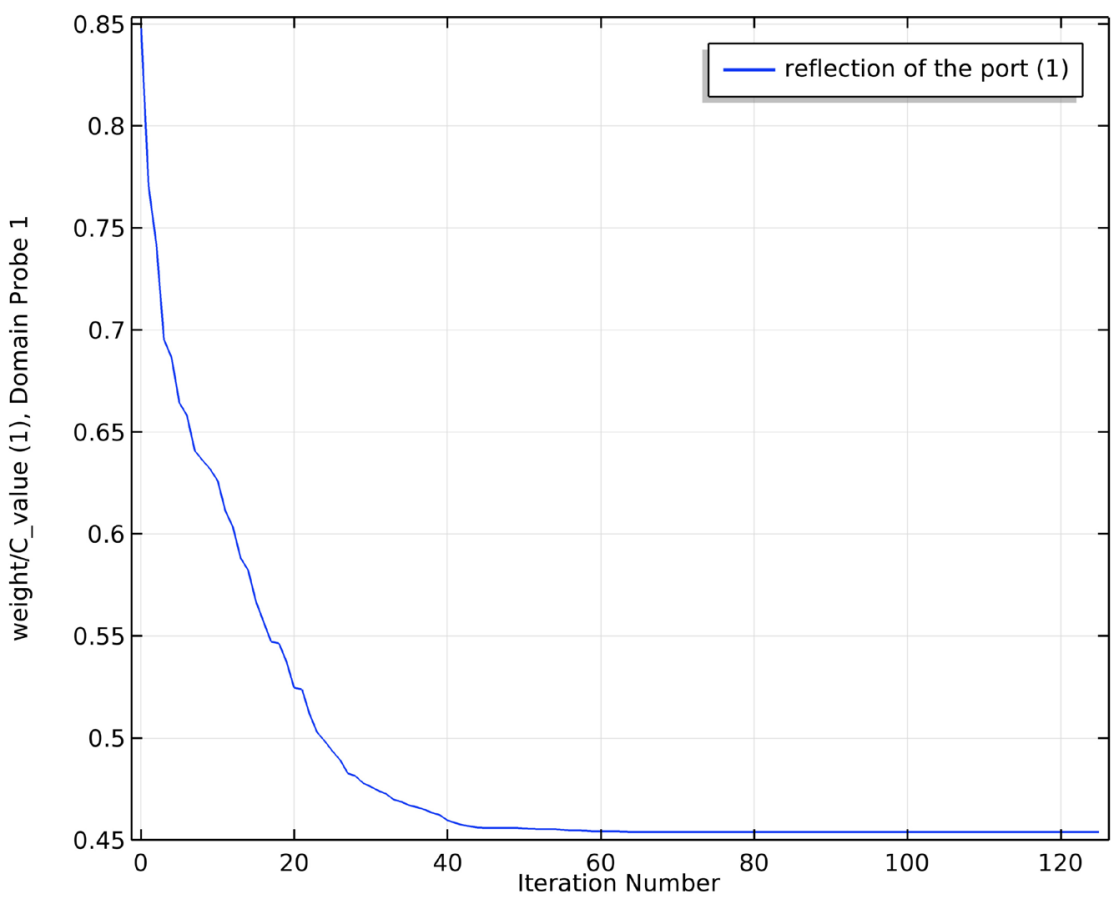

Figure 6. Reflection vs. iteration number.

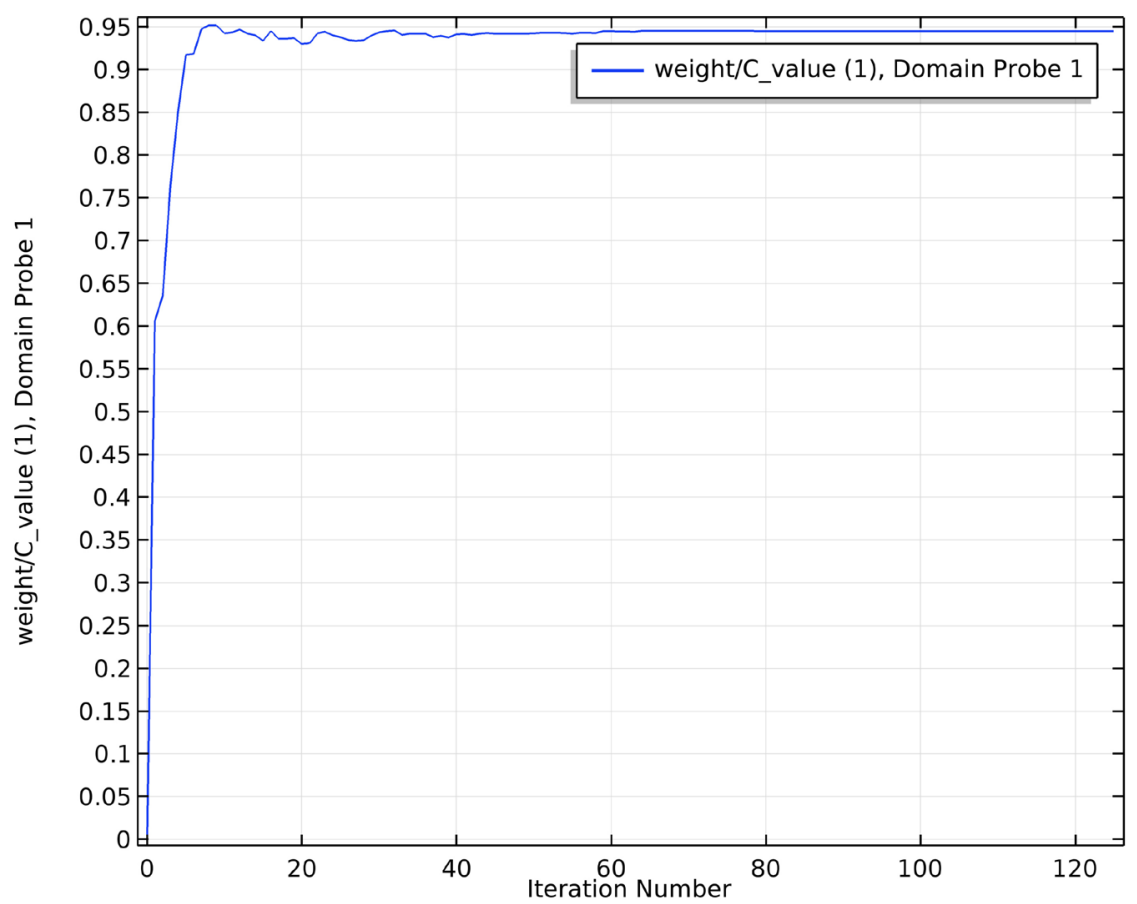

Figure 7. Weight/Area vs. iteration number.

optimization. As the optimization iterated, reflection drops and approaches 0.45 , Weight/Area increases and approaches 0.94, and Objective approaches 105. Even though the reflection was still high in the end, the polar graph demonstrates that the power was indeed concentrated on the desired direction, as shown in Figure 9. 


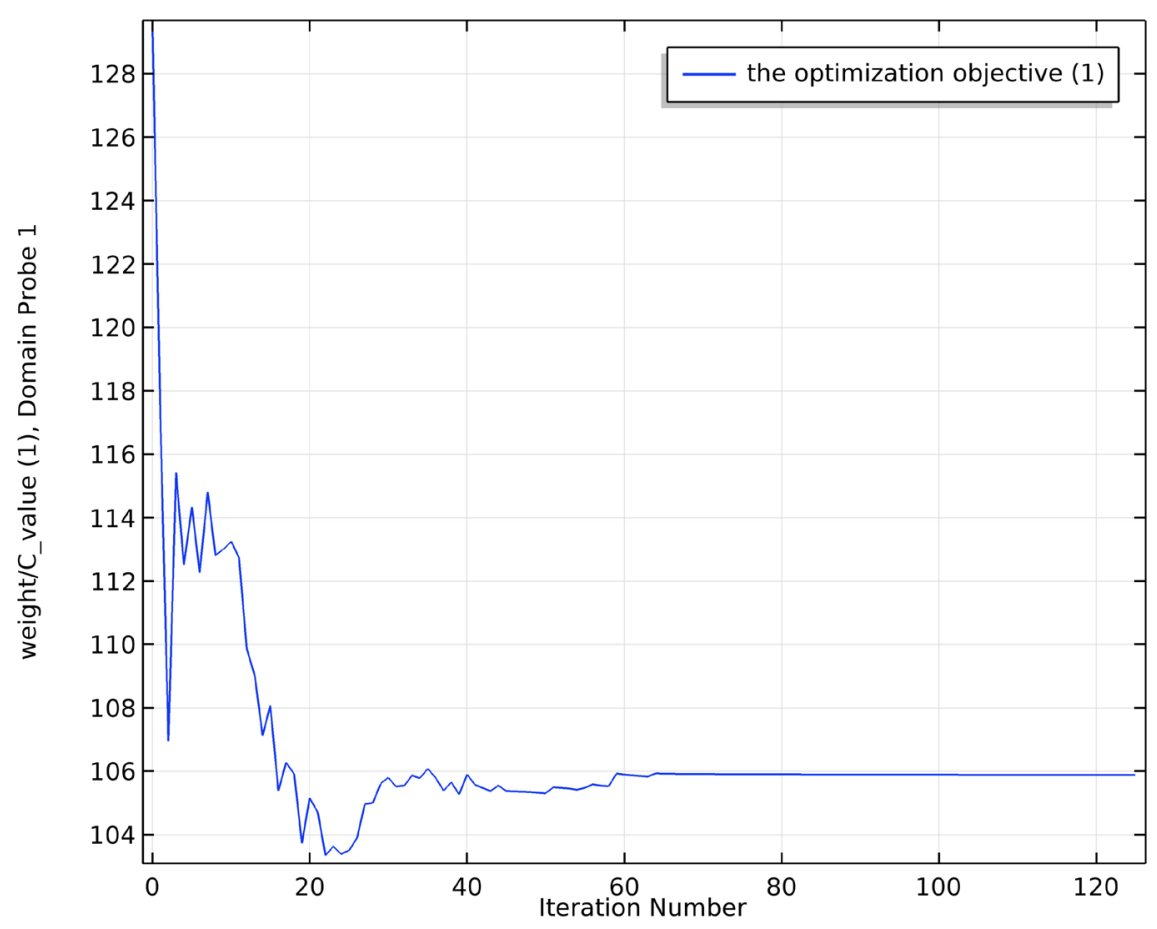

Figure 8. Objective vs. iteration number.

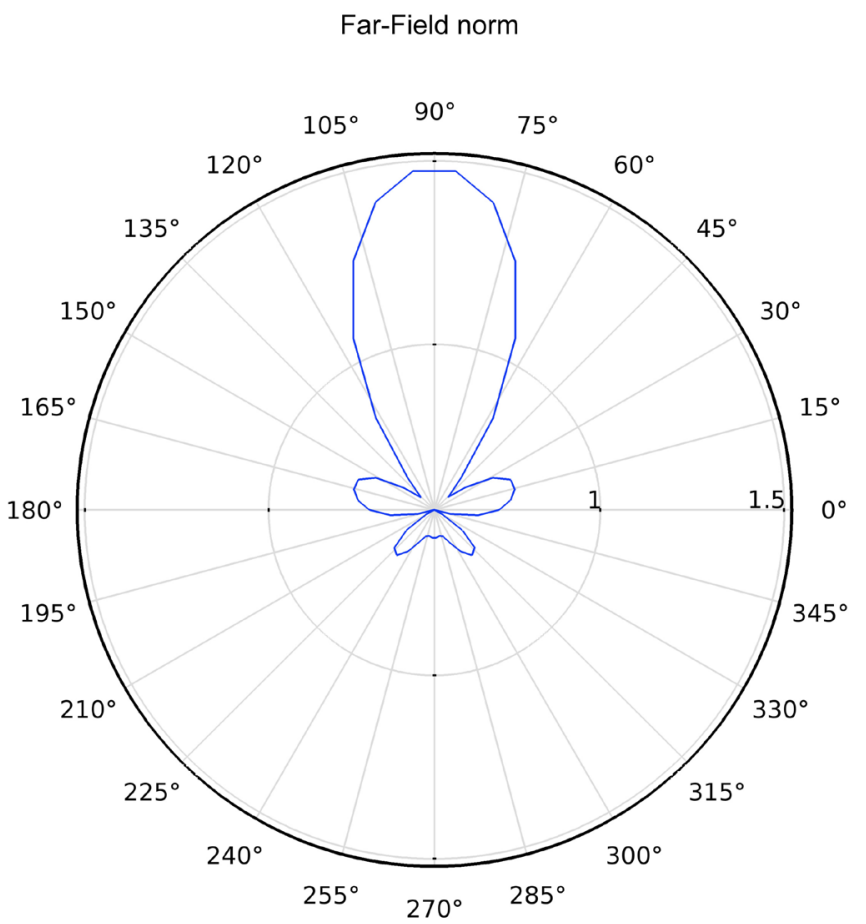

Figure 9. Far field projection of the antenna after topology optimization.

\section{Conclusion}

The topology optimization shows that a distribution of strips of air and polyimide occurring alternatingly provides a high gain for the horn antenna in this study. The materials distribution is non-intuitive, opening new ways for antenna 
performance optimization. This method could be developed to more complicated scenarios, such as phase arrays. Further researches can be conducted to find more combination of materials which may yield even better results.

\section{Conflicts of Interest}

The author declares no conflicts of interest regarding the publication of this paper.

\section{References}

[1] Balanis, C.A. (2016) Antenna Theory: Analysis and Design. John Wiley \& Sons, Hoboken, New Jersey.

[2] Mailloux, R.J. (2017) Phased Array Antenna Handbook. Artech House.

[3] Eggleston, M.S., Messer, K., Zhang, L., Yablonovitch, E. and Wu, M.C. (2015) Optical Antenna Enhanced Spontaneous Emission. Proceedings of the National Academy of Sciences, 112, 1704-1709. https://doi.org/10.1073/pnas.1423294112

[4] Karim, T., Hirokawa, J., Oogimoto, K., Nagatsuma, T., Seto, H., Inoue, I. and Saito, M. (2016) Corporate-Feed Slotted Waveguide Array Antenna in the 350-GHz Band by Silicon Process. IEEE Transactions on Antennas and Propagation, 65, 217-225. https://doi.org/10.1109/TAP.2016.2631132

[5] Lin, Y. and Wang, H. (2016) A Low Phase and Gain Error Passive Phase Shifter in $90 \mathrm{~nm}$ CMOS for $60 \mathrm{GHz}$ Phase Array System Application. In 2016 IEEE MTT-S International Microwave Symposium (IMS), San Francisco, California, May 2016, $1-4$.

[6] Sheta, A. and Mahmoud, S.F. (2008) A Widely Tunable Compact Patch Antenna. IEEE Antennas and Wireless Propagation Letters, 7, 40-42.

https://doi.org/10.1109/LAWP.2008.915796

[7] Tong, J., Muthee, M., Chen, S., Yngvesson S.K. and Jun, Y. (2015) Antenna Enhanced Graphene THz Emitter and Detector. Nano Letters, 15, 5295-5301. https://doi.org/10.1021/acs.nanolett.5b01635

[8] Honeycutt, L. (1998) Communication and Design Course. http://dcr.rpi.edu/commdesign/class1.html

[9] Traviss, D.J., Schmidt, M.K., Aizpurua, J. and Muskens, O.L. (2015) Antenna Resonances in Low Aspect Ratio Semiconductor Nanowires. Optics Express, 23, 22771-22787. https://doi.org/10.1364/OE.23.022771

[10] Zhu, J., Zhang, W. and Xia, L. (2016) Topology Optimization in Aircraft and Aerospace Structures Design. Archives of Computational Methods in Engineering, 23, 595-622. https://doi.org/10.1007/s11831-015-9151-2 